\title{
SÍNDROME DO DESCONFORTO RESPIRATÓRIO AGUDO
}

\author{
ACUTE RESPIRATORY DISTRESS SYNDROME
}

Fabíola Paula Lovetro Galhardo \& José Antônio Baddini Martinez²

\begin{abstract}
${ }^{1}$ Pós-graduanda. ${ }^{2}$ Docente. Divisão de Pneumologia. Departamento de Clínica Médica. Faculdade de Medicina de Ribeirão Preto - USP. CORRESPONDÊNCIA: Prof. Dr. José Antônio Baddini Martinez, Avenida Bandeirantes, 3900 - CEP 14098-900, Ribeirão Preto - SP. Telefone: 016-6022531 - FAX: 016-6366695 - email: jabmarti@fmrp.usp.br
\end{abstract}

GALHARDO FPL \& MARTINEZ JAB. Síndrome do desconforto respiratório agudo. Medicina, Ribeirão Preto, 36: 248-256, abr./dez. 2003.

RESUMO - A Síndrome do Desconforto Respiratório Agudo (SDRA) é um quadro de insuficiência respiratória aguda, devido a intensa resposta inflamatória pulmonar, que ocorre frente a agentes agressores diversos. A agressão pulmonar pode ser local, como nas pneumonias ou aspiração gástrica, ou pode ocorrer por via sistêmica, como, por exemplo, nas septicemias. Apesar de o edema pulmonar por alteração da permeabilidade alveolocapilar ser uma das principais características de suas fases iniciais, na fase proliferativa da síndrome, o acúmulo de diferentes tipos celulares pode levar à deposição de matriz extracelular e à fibrose. Não existe tratamento específico para a SDRA, e as medidas terapêuticas atuais baseiam-se no suporte ventilatório e no tratamento da condição desencadeadora. Um número expressivo de doentes com quadro de remodelamento e proliferação fibrótica pulmonar podem beneficiar-se do uso de esteróides em altas doses. Apesar de ter sido observada redução da mortalidade associada à SDRA, nos últimos anos, devido, basicamente, à melhoria do cuidado intensivo e a novas estratégias ventilatórias, a mortalidade ainda é alta, situando-se em torno de $40 \%$.

UNITERMOS - Síndrome do Desconforto Respiratório Agudo. Insuficiência Respiratória.

A Síndrome de Desconforto Respiratório Agudo (SDRA) é uma síndrome clínica comum, grave, caracterizada por lesão pulmonar aguda, que afeta tanto pacientes clínicos quanto cirúrgicos, adultos ou crianças $^{(1)}$. Descrita, inicialmente, por Ashbaugh e Petty, em 1967, desde então, sua definição e descrição foram redefinidas para melhor incorporação do espectro clínico da doença, bem como para melhor correlação da definição com a fisiopatologia da síndrome.

Em 1994, uma nova definição foi estabelecida pelo American-European Consensus Conference Comittee, a qual foi amplamente adotada por clínicos e pesquisadores da área na identificação da SDRA. ${ }^{(2,3)}$ O consenso reconhece que a gravidade da lesão pulmonar varia, incorporando o conceito de existência de um espectro no desenvolvimento da doença: conside- ra-se que pacientes com hipoxemia menos grave tenham Lesão Pulmonar Aguda (acute lung injury) e aqueles com hipoxemia mais grave tenham Síndrome do Desconforto Respiratório Agudo. ${ }^{(1,2)} \mathrm{O}$ termo SDRA refere-se ao estágio mais grave do espectro da lesão pulmonar aguda. ${ }^{(2,3)}$

Segundo aquele consenso, lesão pulmonar aguda foi conceituada como uma síndrome caracterizada por inflamação pulmonar aguda e persistente, com edema pulmonar devido ao aumento da permeabilidade vascular, associada a três componentes:

1. infiltrado radiológico alveolar bilateral;

2. relação entre a pressão parcial de oxigênio arterial e a fração inspirada de oxigênio $\left(\mathrm{PaO}_{2} / \mathrm{FiO}_{2}\right)$ entre 201 e $300 \mathrm{mmHg}$, independentemente do valor da pressão positiva expiratória final (PEEP); 
3. ausência de evidência clínica de elevação da pressão atrial esquerda, se avaliada, a pressão de enchimento capilar pulmonar não deve exceder $18 \mathrm{mmHg}$.

A síndrome do desconforto respiratório agudo, por sua vez, apresenta a mesma definição da lesão pulmonar aguda, exceto pela presença de hipoxemia mais grave, identificando-se uma relação entre $\mathrm{PaO}_{2} /$ $\mathrm{FiO}_{2}$ igual ou menor a $200 \mathrm{mmHg}$, independentemente do valor da PEEP.

Por definição, a SDRA é um evento agudo, instalando-se, tipicamente, num período entre seis a quarenta e oito horas, persistindo por dias a semanas. ${ }^{(4,5)}$ A doença é, habitualmente, progressiva, caracterizada por estágios variáveis, com manifestações clínicas, histopatológicas e radiográficas específicas. Para o seu diagnóstico, fatores de risco para o desenvolvimento da SRDA devem ser identificados e doenças pulmonares parenquimatosas, difusas, de curso subagudo ou crônico (como sarcoidose ou fibrose pulmonar idiopática) devem ser excluídas, mesmo que possam apresentar alguns elementos constantes na definição da síndrome.

\section{EPIDEMIOLOGIA}

A incidência atual, tanto da lesão pulmonar aguda quanto da síndrome do desconforto respiratório agudo, não é, ainda, completamente conhecida. Estimativa do National Institute of Health (NIH) sugere a incidência anual da SDRA, nos Estados Unidos, próxima a 75 por 100.000 habitantes. ${ }^{(1)}$ Outros estudos mais recentes relataram incidência, variando de 1,5 a 8,3 pacientes para cada 100.000 habitantes. ${ }^{(1,6)}$ Aproximadamente, 10 a $15 \%$ dos pacientes admitidos em uma unidade de terapia intensiva, e cerca de $20 \%$ dos pacientes sob ventilação mecânica, enquadramse nos critérios estabelecidos da SDRA. ${ }^{(7,8)}$ Outros estudos estão em andamento, para melhor determinação da incidência da síndrome.

\section{CAUSAS E CONDIÇÕES PREDISPONENTES}

Mais de sessenta causas da SDRA já foram identificadas. Os fatores mais comumente associados com o desenvolvimento da síndrome podem ser divididos entre aqueles que promovem lesão pulmonar direta e aqueles que causam lesão pulmonar indiretamente, no contexto de um processo sistêmico (Tabela I). ${ }^{(4,5)}$

Sépsis é a causa mais comum da síndrome do

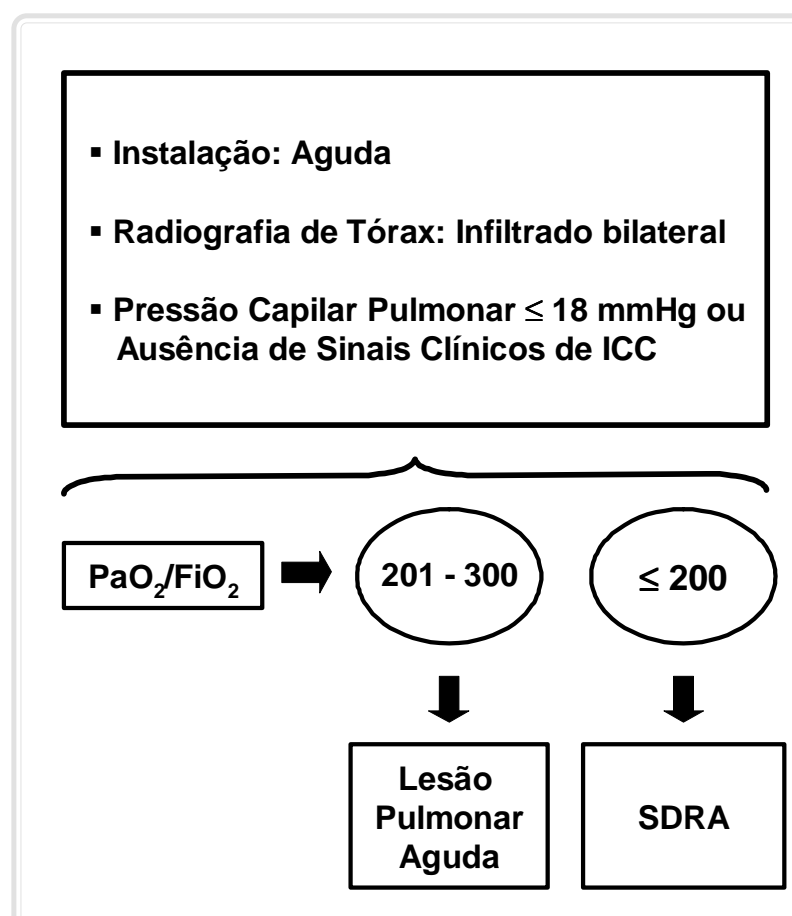

Figura 1 - Critérios diagnósticos para lesão pulmonar aguda e síndrome do desconforto respiratório agudo.

desconforto respiratório agudo. Deve ser, sempre, considerada nos pacientes que desenvolvem a síndrome em associação com febre, hipotensão ou predisposição clínica para infecção grave. De modo geral, sépsis está associada com risco de progressão para lesão pulmonar aguda ou SDRA, em aproximadamente $40 \%$ dos pacientes. ${ }^{(4,5)} \mathrm{O}$ risco de desenvolvimento da SDRA é maior em pacientes sépticos com história de alcoolismo. ${ }^{(9)}$

Aproximadamente, um terço dos pacientes hospitalizados, que aspiram conteúdo gástrico, subseqüentemente, desenvolvem a síndrome. Aparentemente, o $\mathrm{pH}$ baixo, as enzimas gástricas e as partículas de alimentos contribuem para a lesão pulmonar. ${ }^{(4,10)}$ A pneumonia é, provavelmente, a causa mais comum de síndrome do desconforto respiratório agudo, de desenvolvimento fora do ambiente hospitalar. ${ }^{(1)}$ A hemotransfusão de mais de quinze hemoconcentrados também é um fator de risco importante para o desenvolvimento de SDRA, mesmo na ausência de trauma. ${ }^{(5)} \mathrm{O}$ quadro também pode resultar de overdose de várias drogas, incluindo aspirina, cocaína, opióides, fenotiazídicos e antidepressivos tricíclicos. Reações idiossincrásicas também podem ocorrer com certas drogas, como quimioterápicos, nitrofurantoína e contraste radiológico. ${ }^{(12)}$ 
Tabela I - Causas associadas ao desenvolvimento da SDRA

Lesão pulmonar direta

\section{CAUSAS COMUNS}

- Pneumonia

- Aspiração do conteúdo gástrico

\section{CAUSAS MENOS COMUNS}

- Contusão pulmonar

- Embolia gordurosa ou gasosa

- Lesão por inalação

- Edema de reperfusão pós transplante pulmonar ou embolectomia

- Afogamento

\section{Lesão pulmonar indireta}

\section{CAUSAS COMUNS}

- Sépsis

- Trauma grave com choque ou múltiplas transfusões

CAUSAS MENOS COMUNS

- Circulação extracorpórea

- Toxicidade por drogas

- Pancreatite aguda

- Hemotransfusão

- Queimaduras graves

- Múltiplas fraturas

- Pós-transplante de medula óssea

- Causa neurogênica
A presença de múltiplos fatores de risco eleva, substancialmente, o risco do desenvolvimento de SDRA, bem como a presença de condições associadas, como doença pulmonar crônica e baixo pH sanguíneo. ${ }^{(5)}$ Desconforto respiratório agudo, de causa neurogênica deve ser especialmente considerado, em pacientes com sangramento intracraniano ou convulsão. ${ }^{(13)}$

Vários mecanismos podem contribuir para o desenvolvimento da SDRA após trauma ou queimaduras. ${ }^{(4,5)}$ A contusão pulmonar bilateral é um importante fator fisiopatogênico após trauma fechado sobre o tórax. ${ }^{(14)}$ A embolia gordurosa pulmonar, após fratura de ossos longos, também pode promover a síndrome, que, geralmente, ocorre 12 a 48 h após a lesão óssea. ${ }^{(1,4,5)}$ A lesão traumática extensa de tecidos também pode predispor ao desenvolvimento de SDRA, mesmo na ausência de infecção. ${ }^{(1,4,5)}$

\section{PATOGÊNESE}

No aspecto pulmonar, dois componentes distintos constituem a chamada barreira alveolocapilar, o endotélio microvascular e o epitélio alveolar ${ }^{(1)}$. Para a normalidade da função pulmonar, há necessidade de alvéolos patentes, intimamente associados a capilares adequadamente perfundidos. O capilar pulmonar normal apresenta endotélio seletivamente permeável, onde as proteínas permanecem no intravascular, enquanto os fluidos atravessam as membranas, como resultado do balanço entre forças hidrostáticas e gradientes oncóticos. O balanço entre as forças hidrostáticas e oncóticas permite, habitualmente, que pequenas quantidades de fluido extravasem para o espaço intersticial pulmonar. Porém, três mecanismos existem, para impedir a formação de edema intersticial ${ }^{(1)}$ :

- Proteínas intravasculares mantêm um gradiente osmótico favorável à reabsorção de líquido;

- os linfáticos intersticiais podem drenar grande quantidade de líquido para a circulação;

- junções intercelulares firmes, entre as células epiteliais alveolares, previnem o enchimento dos espaços aéreos.

Admite-se que, na SDRA, ocorra lesão do epitélio alveolar e do endotélio capilar, por diferentes mediadores pró-inflamatórios, particularmente pelas citocinas, tais como o fator de necrose tumoral- $\alpha$ (TNF- $\alpha$ ), interleucina 1 e interleucina 8 , liberadas em resposta a grande variedade de precipitantes (lesão pulmonar direta ou indireta). Os pulmões são, particularmente vulneráveis a lesões inflamatórias, porque os mediadores são liberados na circulação e os pulmões recebem a totalidade do débito cardíaco. ${ }^{(15)}$ Como consequiência, neutrófilos são atraídos, tornam-se ativados, liberando mediadores inflamatórios, como oxidantes e proteases que lesam diretamente o epitélio alveolar e o endotélio vascular, propagando o processo 
inflamatório. ${ }^{(1)}$ Desse modo, as barreiras habitualmente responsáveis por impedir o edema alveolar são perdidas, havendo escape de proteínas do espaço intra-vascular em direção ao espaço intersticial, edema intersticial e alveolar. O influxo de líquido rico em proteínas para o interior dos alvéolos altera a integridade do surfactante pulmonar, com lesão adicional ao tecido pulmonar e colapso alveolar. ${ }^{(1)}$

Como conseqüência da agressão pulmonar, da instalação de edema e de microatelectasias, inúmeras alterações fisiopatológicas se estabelecem. ${ }^{(1,2,3)} \mathrm{Ex}$ pressivas reduções da complacência pulmonar levam ao aumento do trabalho muscular respiratório e, também, contribuem para a dispnéia. Alterações da relação ventilação/perfusão e extensas áreas de shunt direito/esquerdo são responsáveis pela hipoxemia acentuada. Hipertensão pulmonar ocorre secundariamente a hipoxemia e, provavelmente, também, pela ação de mediadores inflamatórios circulantes. Essa alteração costuma agravar-se nos indivíduos sob ventilação mecânica com pressão positiva.

\section{PATOLOGIA}

Histologicamente, a lesão pulmonar, na síndrome do desconforto respiratório agudo, é caracterizada como dano alveolar difuso (DAD). ${ }^{(16)}$ Muito em- bora o edema pulmonar seja elemento característico das fases iniciais da doença, o padrão da lesão envolve três estágios patológicos distintos:

- fase exsudativa: caracterizada por edema intersticial e alveolar, bem como pela formação de membranas hialinas; ocorre na primeira semana de evolução do quadro;

- fase proliferativa: caracterizada pela resolução do edema pulmonar, pela proliferação de pneumócitos do tipo II, infiltração intersticial por miofibroblastos e deposição de colágeno;

- fase fibrótica: ocorre em pacientes com doença prolongada, caracterizada por alteração da arquitetura pulmonar normal, fibrose difusa e formação de cistos.

É importante salientar que as três fases histológicas da lesão e reparo não necessariamente ocorrem em todos os pacientes com SDRA. Em muitos indivíduos, o processo inflamatório é autolimitado e a barreira alveolocapilar é rapidamente restabelecida. Em outros, o processo é mais demorado, mas, também, acaba ocorrendo recuperação pulmonar.

No processo de resolução da SDRA, há importante papel para os mecanismos de clearance do edema alveolar. ${ }^{(17,18)}$ Através deles, o edema alveolar é reabsorvido para o interstício, devido ao desenvolvi-




mento de gradientes osmóticos pela ação de canais de sódio, e, possivelmente, cloro, localizados nas membranas de pneumócitos do tipo II. A reabsorção do edema, por sua vez, ocorreria através de poros dos pneumócitos do tipo I. Estudos, em animais e em humanos, têm demonstrado que o clearance do edema alveolar está reduzido na maioria dos pacientes com SDRA, mesmo em fases iniciais da lesão, o que não ocorre em indivíduos com edemas hidrostáticos, como o da insuficiência cardíaca esquerda. ${ }^{(17,18)}$ A presença de grandes prejuízos desse clearance está asso-ciada a pior sobrevida em pacientes com SDRA. Proteínas solúveis, também, poderiam ser reabsorvidas para o interstício através do epitélio pulmonar. Já as proteínas insolúveis, tais como os componentes das membranas hialinas, dependeriam, para sua retirada, da endocitose, a partir das células epiteliais e da fagocitose, a partir dos macrófagos alveolares. A proliferação e a diferenciação dos pneumócitos tipo II são de fundamental importância para a regeneração e a recuperação das estruturas alveolares. Entretanto, em alguns casos, por razões ainda não completamente conhecidas, a inflamação progride, acompanhada pela infiltração local de miofibroblastos, o que resulta no desenvolvimento de fibrose pulmonar progressiva $a^{(1,16)}$.

\section{MANIFESTAÇÕES CLÍNICAS}

O quadro clínico tende a se instalar decorridas horas até dois dias do evento precipitante. A fase aguda é caracterizada por insuficiência respiratória de início agudo, em paciente com condição predisponente para o desenvolvimento da SDRA. ${ }^{(1,2,19)}$ Nesse momento grave hipoxemia, refratária ao tratamento com oxigênio suplementar, estabelece-se. Os pacientes, habitualmente, queixam-se de dispnéia, podendo, igualmente, estar presente tosse seca. Ao exame físico, taquipnéia, tiragem, uso da musculatura acessória da respiração, cianose e estertores difusos são achados comuns. $\mathrm{O}$ achado radiológico característico é a presença de infiltrado alveolar difuso e bilateral, com broncograma aéreo, podendo ocorrer, ainda, derrame pleural. (Figura 3)
Os achados laboratoriais são inespecíficos, podendo incluir leucocitose, evidências compatíveis com coagulação intravascular disseminada, acidose láctica, hipoxemia arterial e aumento do gradiente alveoloarterial de oxigênio. Na grande maioria das vezes, fazse necessária a instalação de ventilação mecânica.

A síndrome pode ser acompanhada de manifestações sistêmicas e falência de outros órgãos, incluindo o sistema nervoso central, rins, fígado, sistema hematopoiético e cardiovascular. Caso venha a ser realizada uma tomografia computadorizada de tórax, será evidenciado preenchimento alveolar, consolidação e atelectasias intercaladas com áreas relativamente poupadas, acometendo, principalmente, as zonas dependentes da gravidade. ${ }^{(1,19)}$

$\mathrm{Na}$ evolução do quadro, a oxigenação tende a melhorar assim que ocorrer a resolução do edema pulmonar. Entretanto, mesmo assim, a maior parte dos pacientes permanece em ventilação mecânica pela persistência da hipoxemia, necessidade de altos volumes/ minutos e baixa complacência pulmonar. Os infiltrados radiológicos, também, podem tornar-se menos densos, refletindo a resolução do edema pulmonar, enquanto as marcas intersticiais permanecem. É a fase em que pode ocorrer o desenvolvimento de enfisema intersticial e de cistos pulmonares. Fibrose pode ocorrer na fase proliferativa refletindo-se como hipertensão pulmonar e faveolamento, ao exame radiológico de tórax.

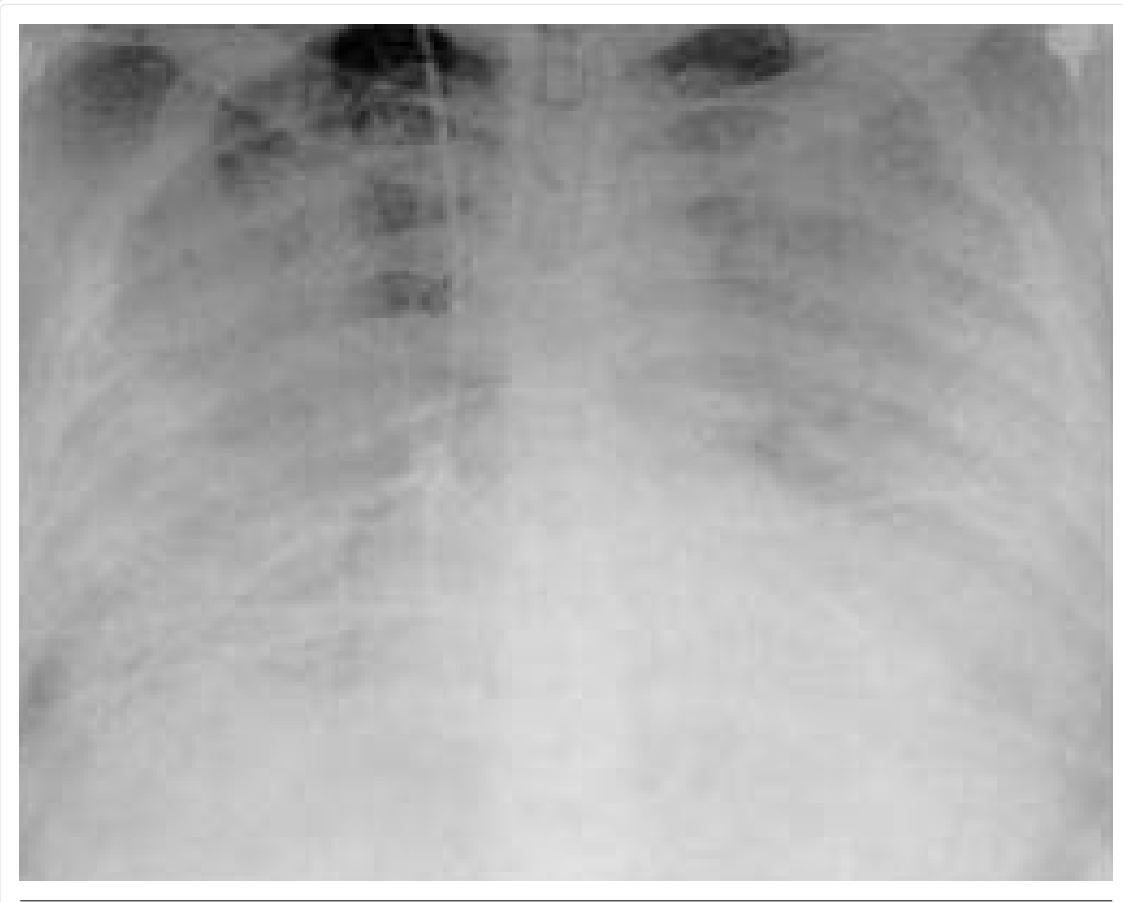

Figura 3 - Radiografia de tórax representativa da SDRA. 


\section{DIAGNÓSTICO DIFERENCIAL}

O edema pulmonar secundário a insuficiência cardíaca congestiva, assemelha-se, clinica e radiologicamente, à SDRA. Desse modo, o passo inicial, no diagnóstico da síndrome, é a determinação da ausência de edema pulmonar cardiogênico. ${ }^{(1,2)}$ A distinção entre ambos, geralmente, é conseguida pela história clínica, pois a distinção radiológica entre as duas entidades, que cursam com infiltrado alveolar bilateral, é difícil. O cateter de Swan-Ganz pode ser utilizado, se o mecanismo de formação do edema não puder ser discernido com confiança ${ }^{(20)}$. Medida de pressão capilar pulmonar, inferior a $18 \mathrm{mmHg}$ favorece lesão pulmonar aguda ou SDRA e não edema pulmonar cardiogênico. Da mesma maneira que o edema pulmonar cardiogênico, várias outras entidades podem ser confundidas com a síndrome do desconforto respiratório agudo. ${ }^{(21)}$

A hemorragia alveolar difusa deve ser considerada, sempre que ocorrer o estabelecimento de insuficiência respiratória aguda em associação a queda da hemoglobina, não explicada de outro modo. A presença de hemoptise pode ser mínima ou ausente previamente à intubação orotraqueal, porém, a avaliação broncoscópica revela secreção sanguinolenta, presente na via aérea durante a hemorragia ativa.

A pneumonia intersticial aguda (Síndrome de Hamman-Rich) é uma forma rara e fulminante de lesão pulmonar, que se instala agudamente (dias a semanas do início dos sintomas), mais comumente em indivíduos previamente sadios. A etiologia e o mecanismo da lesão pulmonar ainda não foram identificados. O diagnóstico é estabelecido pela presença de idiopática síndrome de desconforto respiratório agudo e confirmação anatomopatológica de dano alveolar difuso em organização. O tratamento, habitualmente instituído, é com o uso de corticosteróides em altas doses.

A pneumonia eosinofílica aguda pode evoluir para insuficiência respiratória aguda, ocorrendo em indivíduos previamente hígidos. É caracterizada por tosse, dispnéia, febre e, ocasionalmente, dor torácica. Essa condição pode ser distinguida da SDRA pela presença de grande quantidade de eosinófilos no lavado broncoalveolar (35-55\% das células recuperadas). Não há eosinofilia periférica e existe melhora com o uso de corticosteróides.

A bronquiolite obliterante com pneumonia em organização (BOOP) é, geralmente, caracterizada por um quadro indolente, semelhante ao de pneumonia, associado a tosse, dispnéia e infiltrados alveolares, na radiografia de tórax. ${ }^{(22)}$. Muitos pacientes podem evoluir para quadro de insuficiência respiratória aguda, requerendo intubação e ventilação mecânica. $\mathrm{O}$ tratamento com corticosteróides costuma ser efetivo.

Além de diversas infecções, a tuberculose miliar também deve ser lembrada no contexto do paciente que evolui para insuficiência respiratória aguda secundária a doença febril sistêmica em investigação. ${ }^{(23)}$ Linfomas, leucemias ou disseminação linfangítica de neoplasias sólidas podem promover insuficiência respiratória aguda e comportar-se de maneira semelhante à lesão da SDRA.

\section{TRATAMENTO}

Não existe um tratamento específico para a síndrome do desconforto respiratório agudo. Desse modo, o manejo baseia-se em medidas de suporte e tratamento dirigido para a condição de base subjacente. ${ }^{(1,2,3,19)}$ Atenção especial deve ser dada ao tratamento da causa desencadeante, como, por exemplo, a introdução de antibióticos, para o tratamento de quadros sépticos, ou a abordagem cirúrgica de focos infecciosos abdominais.

A maior parte dos pacientes com SDRA necessita de suporte ventilatório invasivo. O modo mais adequado de manejar o ventilador tem sido extensamente debatido desde a descrição inicial da síndrome. $(1,3,24) \mathrm{O}$ conceito de que a própria ventilação mecânica poderia contribuir para o agravamento da lesão pulmonar estimulou intensa procura pelo melhor manejo das pressões e volumes utilizados na ventilação desses pacientes. Nos indivíduos acometidos pela síndrome, a lesão pulmonar é heterogênea, com áreas de pulmão normal, intercaladas com áreas de pulmão atelectásico. Tais áreas de pulmão normal podem ser lesadas, ao serem hiperinsufladas durante a ventilação mecânica.

Um achado de grande importância foi demonstrar que pacientes com SDRA, tratados com menor volume corrente $(6 \mathrm{ml} / \mathrm{kg})$ apresentam redução da mortalidade em torno de $22 \%$, em relação a pacientes tratados com volumes correntes maiores $(12 \mathrm{ml} / \mathrm{kg})$. $^{(24)}$ A ventilação com menores volumes pulmonares associa-se a menores pressões respiratórias e menor risco de lesão pulmonar, induzida ou perpetuada pela ventilação. Entretanto, com freqüência, o emprego de baixos volumes correntes leva a elevações da pressão arterial de gás carbônico, as quais são aceitas, dentro 
de certos níveis, dentro do conceito da hipercapnia permissiva. A utilização de pressão expiratória positiva final (PEEP) consegue, na maior parte dos pacientes, promover uma redução da $\mathrm{FiO}_{2}$, ao recrutar áreas de pulmão colapsado e aumentar o volume da capacidade residual funcional. Embora o debate sobre o PEEP ideal a ser utilizado na SDRA continue, a construção de curvas pressão/volume à beira do leito, individuais para cada paciente, pode ser bastante útil nesse contexto. O PEEP adequado para o paciente deve situarse acima do ponto de inflexão inferior dessa curva.

Quanto à administração de fluidos, ela deve ser adequada para permitir estabilidade hemodinâmica e boa perfusão dos órgãos, porém, deve-se evitar a hiperhidratação que pode agravar o edema pulmonar e a hipoxemia. ${ }^{(1,3,19)}$ Freqüentemente, a estabilidade hemodinâmica só consegue ser mantida com o emprego de aminas vasoativas.

Na SDRA, existe disfunção do surfactante alveolar, o que poderia sugerir um papel para a terapia de reposição do mesmo. Contudo, até o presente momento, nenhum estudo demonstrou melhora da sobrevida de pacientes tratados com reposição de surfactante..$^{(1,2,3)}$ Novas preparações, contendo proteínas recombinantes e novas maneiras de administração do surfactante, incluindo a instilação intratraqueal e o lavado broncoalveolar, estão em investigação.

O uso de óxido nítrico,no tratamento de pacientes com SDRA, visando a melhor adequação das relações ventilação/perfusão, também já foi avaliado e não pode ser recomendado para tratamento de rotina, embora possa ser útil em pacientes com hipoxemia refratária ${ }^{(1)}$.

Os glicocorticóides são, atualmente, utilizados no tratamento da fase fibrosante da doença, com resultados encorajadores. ${ }^{(1,25,26)}$ Um curso rápido de glicocorticóide pode ser considerado como terapia de resgate em pacientes com doença grave, não responsiva. Não existe beneficio desse tipo de terapia, quando usada de maneira preventiva ou, precocemente, no curso do processo.

Outras medidas de ordem geral, igualmente importantes, incluem o suporte nutricional e a profilaxia de sangramento digestivo e dos fenômenos tromboembólicos. ${ }^{(1)}$

\section{COMPLICAÇÕES}

Pacientes com SDRA, mantidos em ambiente de terapia intensiva e sob ventilação mecânica, estão sujeitos a inúmeras complicações. ${ }^{(3)}$ Dentre elas, podem ser citadas as indicadas a seguir.

- Pneumonia nosocomial: complicação muito comum e temida, é importante causa de morbidade e mortalidade. O diagnóstico torna-se difícil em paciente já com quadro clínico e radiográfico de SDRA. Nesse contexto, a coleta de hemoculturas e culturas quantitativas de secreções pulmonares, obtidas por broncoscopia e cateteres especiais, adquire importância.

- Barotraumas: relacionados à ventilação mecânica, com altos volumes correntes ou pressões; compreendem o pneumotórax, o enfísema subcutâneo, o pneumomediastino, o enfisema intersticial e a embolia gasosa.

- Miopatia: associada ao uso de bloqueadores neuromusculares e corticosteróides, pode dificultar tanto o desmame do respirador, como, também, a recuperação da movimentação dos pacientes.

\section{PROGNÓSTICO}

A sobrevida dos pacientes com SDRA melhorou nos últimos anos e a mortalidade atual é estimada em 35 a 40\%. ${ }^{(27,28)}$ Contudo, a mortalidade varia em função dos fatores de risco que levaram ao desenvolvimento da SDRA, com a existência de insuficiência de múltiplos órgãos e com a presença de co-morbidades. Quanto aos sobreviventes, eles apresentam apenas discretas alterações da função pulmonar e, a longo prazo, apresentam-se assintomáticos, na maioria dos casos. ${ }^{(29)}$ Entretanto, a qualidade de vida dos sobreviventes pode se mostrar diminuída em muitos casos por, pelo menos, um ano. ${ }^{(30)}$

\section{CONCLUSÃO}

Ao longo dos últimos anos, tem-se observado um grande progresso no entendimento de diversos aspectos da SDRA, particularmente nos fatos relacionados a sua epidemiologia, patogenia e evolução anatomopatológica. A mortalidade associada à síndrome mostrou redução, devido, em grande parte, a melhoria das condições de cuidado intensivo e às novas estratégias ventilatórias. Pesquisas adicionais acerca de novas abordagens farmacológicas e ventilatórias podem contribuir, ainda mais, para a redução da mortalidade dessa grave condição clínica. 
GALHARDO FPL \& MARTINEZ JAB. Acute respiratory distress syndrome. Medicina, Ribeirão Preto, 36: 248-256, apr./dec. 2003.

ABSTRACT - The Acute Respiratory Distress Syndrome (ARDS) is a syndrome of acute respiratory failure associated to severe pulmonary inflammation secondary to acute lung injury. The injury agent may be local, like pneumonias or aspiration syndromes, or systemic like sepsis. Pulmonary edema secondary to disturbs in alveolar capillary barrier is a hallmark of acute phase but, in the proliferative phase cellular infiltration with pulmonary remodeling may lead to fibrosis in some patients. There is not a specific treatment for ARDS. The present therapeutic interventions are respiratory support and the treatment of the causal subjacent condition. Some patients in the proliferative phase may show good response to high doses of steroids. It has been seen a decrease in ARDS mortality during the last years, probably due to improvements in the quality of intensive care units and new ventilatory strategies. However, ARDS associated mortality is still high, around $40 \%$.

UNITERMS - Acute Respiratory Distress Syndrome. Respiratory Insufficiency.

\section{REFERÊNCIAS BIBLIOGRÁFICAS}

1 - WARE LB \& MATTHAY MA. The acute respiratory distress syndrome. N Engl J Med 342: 1334-1349, 2000.

2 - BERNARD GR; ARTIGAS A; BRIGAM KL; CARLET J; FALKE L; HUDSON L; LAMY M; LEGALL JR; MORRIAA\& SPRAGG R. The American-European Consensus Conference on ARDS. Definitions, mechanisms, relevant outcomes, and clinical trial coordination. Am J Respir Crit Care Med 149: 818-824, 1994.

3 - ARTIGAS A; BERNARD GR; CARLET J; DREYFUSS D; GATTINONI L; HUDSON L; LAMY M; MARINI JL, MATTHAY MA; PINSKY MR; SPRAGG R, SUTER PM, AND THE CONSENSUS COMMITTEE The American-European Consensus Conference on ARDS, Part 2: Ventilatory, pharmacologic, supportive therapy, study design strategies, and issues related to recovery and remodeling. Acute respiratory distress syndrome. Am J Respir Crit Care Med 157: 1332-1347, 1998.

4 - PEPE PE; POTKIN RT; REUS DH; HUDSON LD \& CARRICO CJ. Clinical predictors of the adult respiratory distress syndrome. Am J Surg 144: 124-130, 1982.

5 - HUDSON LD; MILBERG JA; ANARDI D \& MAUNDER RJ. Clinical risks for development of the acute respiratory distress syndrome. Am J Respir Crit Care Med 151: 293-301, 1995.

6 - THOMSEN GE \& MORRIS AH. Incidence of the adult respiratory distress syndrome in the state of Utah. Am J Respir Crit Care Med 152: 965-971, 1995.

7 - BERSTEN AD, EDIBAM C; HUNT T \& MORAN J. Incidence and mortality of acute lung injury and the acute respiratory distress syndrome in three Australian States. Am J Respir Crit Care Med 165: 443-448, 2002.

8 - ESTEBAN A; ANZUETO A; FRUTOS F; ALIA I BROCHARD L; STEWART TE; BENITO S; EPSTEIN SK; APEZTEGUIAC; NIGHTINGALE P;ARROLIGAAC; TOBIN MJ; FOR THE MECHANICAL VENTILATION INTERNATIONAL STUDY GROUP. Characteristics and outcomes in adult patients receiving mechanical ventilation: a 28-day international study. JAMA 287: 345-355, 2002.
9 - MOSS M; BUCHER B; MOORE FA; MOORE EE \& PARSONS $\mathrm{PE}$. The role of chronic alcohol abuse in the development of acute respiratory distress syndrome in adults. JAMA 275: 50-54, 1996.

10 - TIETJEN PA; KANER RJ \& QUINN CE. Aspiration emergencies. Clin Chest Med 15: 117-135, 1994.

11 - BAUMANN WR; JUNG RC; KOSS M; BOYLEN CT; NAVARRO L \& SHARMA OP. Incidence and mortality of adult respiratory distress syndrome: a prospective analysis from a large metropolitan hospital. Crit Care Med 14: 1-4, 1986.

12 - REED CR \& GLAUSER FL. Drug-induced noncardiogenic pulmonary edema. Chest 100: 1120-1124, 1991.

13 - COLICE GL; MATTHAY MA;BASS E \& MATTHAY RA. Neurogenic pulmonary edema. Am Rev Respir Dis. 130: 941948, 1984.

14 - RUSSELL GB \& CAMPBELL DB. Thoracic trauma and the adult respiratory distress syndrome. Semin Thorac Cardiovasc Surg 4: 241-246, 1992.

15. MARTIN TR. Lung cytokines and ARDS. Chest 116: 2S-8S, 1999. Suppl. 1.

16 - TOMASHEFSKI JF Jr. Pulmonary pathology of acute respiratory distress syndrome. Clin Chest Med 21: 435-466, 2000.

17 - MATTHAY MA. Alveolar clearance in patients with ARDS: Does it make a difference? Chest 122: 340 S-343 S, 2002. Suppl. 6.

18 - WARE LB \& MATTHAY MA. Alveolar fluid clearance is impaired in the majority of patients with acute lung injury and acute respiratory distress syndrome. Am J Respir Crit Care Med 163: 1376-1383, 2001.

19 - STEINBERG KP \& HUDSON LD. Acute lung injury and acute respiratory distress syndrome. The clinical syndrome. Clin Chest Med 21: 401-417, 2000. 
20 - MEADE MO; COOK RJ; GUYATT GH; GROLL R; KACHURA JR; BEDARD M; COOK DJ; SLUTSKY AS \& STEWART TE Interobserver variation in interpreting chest radiographs for the diagnosis of acute respiratory distress syndrome. Am J Respir Crit Care Med 161: 85-90, 2000.

21 - SCHWARZ MI \& ALBERT RK. "Imitators" of the ARDS: implications for diagnosis and treatment. Chest 125: 1530-1535, 2004.

22 - NIZAMI IY; KISSNER DG; VISSCHER DW \& DUBAYBO BA. Idiopathic bronchiolitis obliterans with organizing pneumonia. An acute and life-threatening syndrome. Chest 108: 271-277, 1995.

23 - LINTIN SN \& ISAAC PA. Miliary tuberculosis presenting as adult respiratory distress syndrome. Intensive Care Med 14: 672-674, 1988.

24 - THEACUTE RESPIRATORY DISTRESS SYNDROME NETWORK. Ventilation with lower tidal volumes as compared with traditional tidal volumes for acute lung injury and the acute respiratory distress syndrome. N Engl J Med 342: 1301-1318, 2000.

25 - MEDURI GU; CHINNAJ; LEEPER KV; WUNDERINK RG; TOLLEY E;WINER-MURAM HT; KHARE V \& ELTORKY M. Corticosteroid rescue treatment of progressive fibroproliferation in late ARDS. Patterns of response and predictors of outcome. Chest 105: 1516-1527, 1994.
26 - BERNARD GR; LUCE JM; SPRUNG CL; RINALDO JE; TATE RM; SIBBAD WJ; KARIMAN K; HIGGINS S; BRADLEY R \& METZ CA. High-dose corticosteroids in patients with the adult respiratory distress syndrome. N Engl J Med 317: 1565-1570, 1987.

27 - MILBERG JA; DAVIS DR; STEINBERG KP \& HUDSON LD. Improved survival of patients with acute respiratory distress syndrome (ARDS): 1983-1993. JAMA 273: 306-309, 1995.

28 - LUHR OR; ANTONSEN K; KARLSSON M; AARDAL S; THORSTEINSSONA; FROSTELL CG \& BONDE J. Incidence and mortality after acute respiratory failure and acute respiratory distress syndrome in Sweden, Denmark, and Iceland. The ARF Study Group. Am J Respir Crit Care Med 159: 1849-1861, 1999.

29 - NEFF TA; STOCKER R; FREY HR; STEIN S \& RUSSI EW. Long-term assessment of lung function in survivors of severe ARDS. Chest 123: 845-853, 2003.

30 - ORME J Jr; ROMNEY JS; HOPKINS RO; POPE D; CHAN KJ; THOMSEN G; CRAPO RO \& WEAVER LK. Pulmonary function and health-related quality of life in survivors of acute respiratory distress syndrome. Am J Respir Crit Care Med 167: 690-694, 2003. 